\title{
Asymptotic Properties of the Markov Chain Model method of finding Markov chains Generators of Empirical Transition Matrices in Credit Ratings Applications
}

\author{
Fred Nyamitago Monari ${ }^{1}$,Dr. George Otieno Orwa ${ }^{2}$,Dr. Joseph Kyalo \\ Mung'atu ${ }^{3}$ \\ Department of Statistics and Actuarial Science, Jomo Kenyatta University of Agriculture and Technology, \\ Kenya
}

\begin{abstract}
Credit quality changes need to be analysed from time to time. A good model for analysis needs to determine the Capital and reserves needed to support Credit Instruments portfolios as well as individual Credits. Conditions under which the Series method of finding Markov chains generators of Empirical Transition Matrices in Credit Ratings applications are identified in this article. Searching for valid Generators especially when a true generator does not exist and the properties of the series method is shown. Credit exposures Transition from one rating to another as well as Historical information are used to model estimation that provide a description of Credit quality evolution probability. Time Homogeneous Markov Model specification is popularly used but it is only good in providing a description of portfolio risk changes in the short run hence restrictive in long run Credit processes. I propose a test which is simple and is of time homogeneity null hypothesis performed on all types of data reported often. The data used in the test is Sovereign debt, Municipal Bonds and Commercial paper. I find that transitions on municipal Bond ratings are described adequately for a period of up to five years. The Commercial paper assumes Markovian characteristics for a period of up to Six Months on a scale of 30 days transitions and the Sovereign debt transitions are described adequately by the Markov model using small data sample sizes.
\end{abstract}

Keywords: Transition matrix, Generator, Eigen value, Markov

\section{Introduction}

Transition Matrix rating has been receiving increased attention in the financial industry. Publications of annual updates in transition matrices history is published annually in the US by Moody's and Standard and Poor's. In Kenya, there are two major Credit Rating agencies i. e Metropol and Credit Africa who provide the historical ratings of individual borrowers. Models have been proposed that analyse Credit Risk changes and their aim is to determine the Capital and reserves needed to support the Credit instruments portfolios and individual credits. The quality of a Credit is denoted by summarization of ratings in probability of default and default rate loss. Basel ii agreement (2004) made it a requirement for Institutions to provide an asset rating that covers default probability of one Year and the loss expected given the default. Banks and other financial Institutions rely entirely on these models and systems in order to produce stable and accurate risk representations of credit loss for Credit exposures of similar nature for both Current and future populations. In portfolio selection. most financial Institutions use this information as well as historical information on Credit exposures transitions from one rating to another and the information is used for estimation of various models that provide a description of the credit quality evolution. the specification which is popular is the time homogeneous model deemed to be simple. In transition probability terms, this information provides a specification of stochastic process but however it does not address the issue of transitional matrix generators especially in cases where the transitional matrix is unbounded.

\subsection{Seeking a Candidate Generator}

\section{Methodology}

A Markov transition matrix $\mathrm{P}$ i. e $\mathrm{P}$ is an $\mathrm{N} \times \mathrm{N}$ real Matrix with row sums 1 and non- negative entries is non homogeneous. The main interest here is in finding a generator $\mathrm{Q}$ which is also an $\mathrm{N} \times \mathrm{N}$ matrix with row sums 0 and non-negative off diagonal entries such that throughout;

$$
\begin{gathered}
\exp (Q)=P \\
\exp (t Q)=I+t Q+(t Q)^{2}=2 !+(t Q)^{3}=3 !+\ldots
\end{gathered}
$$

\section{2 Identifying a Matrix Generator}


Let $\mathrm{P}$ be a time homogeneous Markov Transition matrix i. e an $\mathrm{N} \times \mathrm{N}$ real Matrix with non-negative entries and with row sums 1 . The main interest is in finding a generator $\mathrm{Q}$ i. e an $\mathrm{N} \times \mathrm{N}$ real matrix having nonnegative off diagonal entries with row-sums 0 and $\exp (Q)=P$.

Throughout hence,

I is the $\mathrm{N} \times \mathrm{N}$ identity Matrix

$$
\exp (t Q)=I+t Q+(t Q) 2=2 !+(t Q) 3=3 !+\ldots
$$

$\mathrm{P}$ is for one year i.e $t=1$.

The starting point of our computation is given by;

$S=\max \left\{(a-1)^{2}+b^{2} ; a+b i\right.$ is an eigen value of $\left.P, a, b \in \mathfrak{R}\right\}$

$\mathrm{S}$ is computed by examining all eigen values of $\mathrm{P}$, of the form $a+b i$ where $\mathrm{a}$ and $\mathrm{b}$ are real, computing the absolute square of eigen value minus 1 i. e $(a-1)+b 2$ and the maximum taken of these absolute squares.

\section{Property 1}

Let $P$ be an $N \times N$ Markov Transition matrix and suppose $S<1$, then series

$$
\tilde{Q}=(P-1)-(P-1)^{2} / 2+(P-1)^{3} / 3-(P-1)^{4} / 4+\ldots
$$

quickly converges geometrically and gives rise to an $N \times N$ Matrix $\mathrm{Q}^{\sim}$ with row sums 0 such that

$$
\exp (\tilde{Q})=P
$$

The proof of this property can be found in Zahl (1955, P 96) following Wedderburn (1934, P 122) result. This theorem is a simple method in terms of matrices summing for obtaining Matrix $\mathrm{Q}$ having all Generator properties.

\section{Property 2}

What if we have all diagonal entries of Transition Matrix $\mathrm{P}$ greater than $\frac{1}{2}\left(P_{i i}>0.5\right)$ for all $i$ ?

$S<1$ hence convergence of series in Property 1 is guaranteed. Matrix P is strictly

dominant (Horn and Johnson (1985), pg 302). This condition is also proved by Cuthbert (1972, 1973), that with this condition, $\mathrm{P}$ can have only one generator at most and if the generator exists, then is is a unique generator. The series will converge if $S<1$ even if some of the diagonal entries of P are less than 0.5.

Condition in Property 2 above denotes that Transition Matrix P is strictly diagonally dominant (Horn and Johnson (1985) pg 302). P can have one Generator at most (Cuthbert $(1972,1973)$ ) and if the generator exists, then it is unique. The condition is a sufficient one in that Series 3.1 may converge and have $s<1$ even when some of the diagonal entries of $\mathrm{P}$ are less than 0.5 .

\subsection{The condition of Non Negativity}

Matrix $\tilde{Q}$ in Property 1 is not always guaranteed to have non-negative off diagonal entries, leads to a Transition Matrix being Unbounded. This is a major setback since if $\tilde{Q}$ has a negative off diagonal entry, then $P_{t}=\exp (t \tilde{Q})$ for sufficient small $t>0$. This implies that $P_{t}$ will not be a proper Markov Transitional Matrix which is unacceptable. Usually the negative off-diagonal entries of $\tilde{Q}^{\sim}$ are usually small. This problem can be corrected by replacing the negative entries with 0 , then add the appropriate value back to the corresponding diagonal entry in order to preserve the property of having row sum 0 . This means that once we have $\widetilde{Q}$ a new matrix Q can be obtained by;

$$
\begin{aligned}
& q_{i j}=\max \left(\tilde{q}_{i j}, 0\right), j \neq i \\
& q_{i i}=\tilde{q}_{i i}+\sum_{j \neq i} \min \left(\tilde{q}_{i j}, 0\right)
\end{aligned}
$$

3.2 was used by Zahl, 1955. The new matrix Q will have the row sums 0 and non-negative off diagonal entries but will not satisfy $\exp (Q)=P$ exactly.

Another method of obtaining $Q$ can be done by adding the negative values to all entries of the same row and not only on the diagonal entry having the correct sign, proportional to their absolute values. Let;

$$
\begin{aligned}
& G_{i}=\left|\widetilde{q}_{i i}\right|+\sum_{j \neq i} \operatorname{Max}\left(\tilde{q}_{i j}, 0\right) \\
& B_{i}=\sum_{j \neq i} \operatorname{Max}\left(-\tilde{q}_{i j}, 0\right)
\end{aligned}
$$


be the "good" and "bad" totals for each row $i$. Set

$q_{i j}=\left\{o, i \neq j\right.$ and $\left.\tilde{q}_{i j}<o \tilde{q}_{i j}-B_{i}\left|\tilde{q}_{i j}\right| / G_{i}\right\}$

otherwise if $\mathrm{Gi}>0$

$\widetilde{q_{i j}}$, otherwise if $G_{i=0}$

Since $\sum_{j} \tilde{q}_{i j}=0$, we will have $G i \geq B i$ hence (3. 3) guarantees that $q_{i j} \geq 0$ for $i$.

If $\tilde{Q}$ has slightly negative off diagonal elements and values $-q_{i i}$ are sufficiently large, (3. 3) and (3. 2) will be fairly similar. The choice of where to add the extra back in the modification of $\mathrm{Q}$ can be done by optimising the choice as a Multivariate functions. However, the improvement rarely makes any substantial difference to the distance of $\exp (Q)$ to $\mathrm{P}$.

If $\widetilde{Q}^{\sim}$ computed in Theorem 1 is not valid, a valid generator still exists and there may exist more than one such valid generators for a given Matrix of P. Certain conditions may also lead to conclusion of nonexistence of a generator for a given transition matrix $\mathrm{P}$ as illustrated in the Property 3 below.

\section{Property 3}

Let $P$ be a Transition Matrix. Suppose that either;

(a) $\operatorname{det}(P) \leq 0$; or

(b) $\operatorname{det}(P)>\pi_{i} p_{i i}$; or

(c) There are states $i$ and $j$ such that $j$ is accessible from $i$ but $p_{i j}=0$

Then exact generator of $P$ does not exist.

\section{Property 4}

Given $P$ to be a transition Matrix;

(a) $P$ will have one generator at most if $\operatorname{det}(P)>1 / 2$

(b) $\log (P)$ is the only generator possible for $P$ if

$\operatorname{det}(P)>\frac{1}{2}$ and $\|P-1\|<\frac{1}{2}$

(c) $\log (P)$ is the only generator possible for $P$ if $P$ has eigen values which are distinct and

$$
\operatorname{det}(P)>e^{-\Pi}
$$

The property below is also observed. The property was also got by Singer and Spiler-man (1976, pp 29-30).

\section{Property 5}

If $P$ is a Transition Matrix having eigen values which are real and distinct;

(a) If $P$ has eigen value which are all positive, then $\log (\mathrm{P})$ has the only real Matrix $Q$ such that $\exp (\mathrm{Q})=\mathrm{P}$

(b) If $P$ has any eigen values which are negative, then there is no real matrix $Q$ such that $\exp (Q)=P$.

Property 4 and 5 further validate the matrix $\widetilde{Q}$ from property 1 which results into the sub property below;

\section{Corollary}

If $P$ is a Transition Matrix, then at least one of the three conditions below hold;

(i) $\operatorname{det}(P)>\frac{1}{2}$ and ||$P-1||<\frac{1}{2}$ or

(ii) $\mathrm{P}$ has eigen values which are distinct and $\operatorname{det}(P)>e-\pi$

(iii) $P$ has real eigen values which are distinct

If Series in Property 1 converges to a matrix $\mathrm{Q}$ which has negative off-diagonal entries, then a valid generator for $\mathrm{P}$ does not exist.

Other eigen values of $\mathrm{P}$ conditions are known which may make it possible to have valid generator $\mathrm{Q}$. A proof by Elving (1937) shows that if $\mathrm{P}$ has a complex eigen value other than 1 or $\mathrm{P}$ has a real eigen value which is negative, then there does not exist a valid generator for P. Runnenberg (1962) further proves that matrix P of $\mathrm{N}$ nature is having a valid generator, then each complex eigen value of $\mathrm{P}$ must be within the complex plane region bearing the boundary curve;

$$
\left\{\left(\exp (-s+s \cos (2 \pi / N)), \exp (-s+s \cos (2 \pi / N)) \sin (s \sin (2 \pi / N)) ; o \leq s \leq \frac{\pi}{\sin (2 \pi / N)}\right\}\right.
$$

In order for $P$ to have a valid generator, each condition above is necessary as shown in property 3 or property 5 (b).

A more quantitative version of property 3 (c) can be shown finally in property 6 below; 


\section{Property 6}

If the generator of $P$ is valid, then the entries of $P$ must satisfy that;

$$
p_{i k} \geq m^{m} r^{r}(m+r)^{-m-r} \sum_{j}\left(p_{i j}-b_{m}\right)\left(p_{j k}-b_{r}\right) 1_{p i j}>b_{m}, p_{j k}>b_{r} \text {, }
$$

$m$ and $r$ are positive integers.

$$
b_{m}=\sum_{\ell=m+1}^{\infty} e^{-\lambda} \lambda^{\ell} / \ell !=1-\sum_{\ell=0}^{m} e^{\lambda} \lambda^{\ell} / \ell !
$$

which is equated to probability $M^{I}>m, N^{I}$ has mean $\lambda=\max \left(-\mathrm{Q}_{\mathrm{ii}}\right)$ and is a Poisson random variable. $1_{B}$ is an indicator function from boolean event $B$.

Note

If generator for $P$ is non existent, still it is possible to have matrix $\mathrm{P}_{1 / 2}$ such that $\left(P_{1 / 2}\right)^{2}=P$. Kingman(1962) showed that whether $P$ is non singular and all positive integers $n$ have matrix

$\mathrm{P}_{1 / \mathrm{n}}$ such that $\left(P_{1 / n}\right)^{n}=P$, then the generator for $\mathrm{P}$ exists.

\section{Searching for a Valid Generator}

Suppose Series 3. 1 does not converge or converges to matrix having terms which are negative offdiagonal, still it is possible to have a generator. Theoretically finding this generator is possible by having to check the logarithm function and computing $\log (P)$ for every branch of the logarithm function and checking time to time that the non negativity condition is satisfied. If the eigen values of $P$ are distinct, then it leads to property 7 below.

\section{Property 7}

If eigen values of $P$ are distinct and $Q$ a generator of $P$, then each eigen value denoted as $\lambda$ of $Q$ satisfies that $\left|\tau_{\mathrm{m}} \lambda\right| \leq|\ln (\operatorname{det}(\mathrm{P}))|$. A finite number in particular of $\log (P)$ branch values could be the possible generators of $P$. It is important to note that from property 3 (a), no generator will exist if $\operatorname{det}(P) \leq 0$. This Theorem makes it possible to have a finite logarithm or construct the same finite logarithm whenever $\mathrm{P}$ has eigen values which are distinct and all possible generators of $P$ can be searched. Lagrange interpolation can be used; if $P$ is $n \times n$ with eigen values which are distinct $r_{1}, r_{2}, r_{3}, \ldots, r_{\mathrm{n}}$ and $f$ is any function analytic in the neighbourhood of each eigen value, then $f(P)=g(P), g$ is the polynomial degree $n-1$ such that $g\left(r_{j}\right)=f\left(r_{j}\right)$ for each j.

According to Sylvester's formula E. F Singer and Spiderman (1976), the Lagrange interpolation formula says that;

$$
g(x)=\sum_{j} \Pi_{k \neq j} \frac{x-r_{k}}{r_{j}-r k} f\left(r_{j}\right) \ldots
$$

From equation 3.4 above, the sum is on all eigen values $\mathrm{rj}$ and product is on all eigen values $r_{k}$ with the exception of $r_{j}$.

For one to look or search for generators values $f\left(r_{j}\right)$, each of them should be equal to logarithm of $r_{j} . r_{j}$ can be a complex number and $f\left(r_{j}\right)$ values satisfy;

$$
f\left(r_{j}\right)=\log \left|r_{j}\right|+i\left(\operatorname{Arg}\left(r_{j}\right)+2 \pi k_{j}\right)
$$

Integers $k_{j}$ are subject to property 7 condition. Using Lagrange interpolation, $f(P)$ can be computed for each $k_{1}$, $k_{2}, \cdots, k_{n}$ and monitoring the non-negative off diagonal condition for $f(P)$ which may arise.

Property 7 can be summarised in the following steps,

Step 1 Compute and verify that the eigen values $r_{1}, r_{2}, \cdots, r_{n}$ of $P$ are all distinct.

Step 2 For each eigen value $r_{j}$, integer $k$ is chosen such that;

$$
\lambda=\log \left|r_{j}\right|+i\left(\operatorname{Arg}\left(r_{j}\right)+2 \pi k_{j}\right)
$$

satisfies restriction of Property 7 such that;

$\left|\tau_{\mathrm{m}} \lambda\right|=\mid \operatorname{Arg}\left(r_{j}+2 \pi k_{j}|\leq| \ln (\operatorname{det}(P)) \mid\right.$

Step 3 For integers $k_{1}, k_{2}, \cdots, k_{n}$, 
$\mathrm{f}\left(r_{j}\right)=\log \left|r_{j}\right|+i\left(\operatorname{Arg}\left(r_{j}\right)+2 \pi k_{j}\right)$

is set and $g(x)$ is the function from 3.4

Step 4 Matrix $Q=g(P)$ is computed for the function $g(x)$ to verify whether it is a valid generator.

Step 5 Step 2 is repeated by modifying one or more $k_{j}$ until all $k_{1}, k_{2}, \cdots, k_{n}$ have been taken into consideration. The above procedure is sufficient since in practise, the Credit rating Transition Matrices will most likely have distinct eigen values. The search will be more intense in rare cases where there are repeated eigen values.

\section{4 Markov Chain Model}

The Markov chain model due to its relative simplicity is used increasingly by financial In- stitutions and practitioners. In the Markov chain model which is simple and discrete, the stochastic state process $X_{t}$ at discrete time $t$ forms a finite or countable set. The convenient way is to provide a label of the positive integers states. The unique number of finite states is the Markov chain denoted by the integer $k$ and $P_{i j}(t)$ denotes the one step transition probability $x_{i+1}=j$ given $x_{t}=i$. The Transitional probabilities function of initial and final states is emphasized by the above representation as well as the transitional time. Transitional probabilities which are stationery in Markov process are mostly independent of the time variable. Hence in such cases, it can be denoted $P_{i j}\left(t=P_{i j}\right)$. Organization of all transitional probabilities is of the form,

$P=\left(\begin{array}{cccc}P_{11} & P_{12} & \ldots & P_{1 k} \\ P_{21} & P_{22} & \ldots & P_{2 k} \\ \ldots & \ldots & \ldots & \ldots \\ P_{k 1} & P_{k 2} & \ldots & P_{k k}\end{array}\right)$

The transitional probabilities for each row is equal to one i. e

$\sum_{j=1}^{k} P_{i j}=1$

The state may move to any one of the alternative states or with probability Pii remain unchanged. Markov process evolution probability across the states in all sets possible can be represented as;

$X_{t+1}=X_{t} P$

The above equation is simple thus making the Markov Chain Model to be attractive to those researching on Credit transition behaviour. Identifying single asset risk category Xt e. $\mathrm{g}$

$X_{t}=(0,0,1.0, \ldots, 0)$

indicates the asset being in the third risk group. then;

$X_{\mathrm{t}+1}=\left(p_{31}, p_{32}, \ldots, p_{3 k}\right)$

at time $t$, is the asset probability distribution in risk state 3 . Alternatively, in time $t+1, x_{t+1}$ is the corresponding distribution of the distribution $x t$ of assets across time $t$ risk categories and across the whole portfolio, the framework probability applies. The transition equation;

$x_{t+1}=x_{t} p_{t}$

is for the non-homogeneous case and $\mathrm{t}, \mathrm{Pt}$ is the index for the probability transition matrix. This is Markovian model since next period distribution states depend on the current period distribution and not on the previous period distribution. The current state is mostly relied irrespective of the history. This means that the likelihood of a credit moving to another level of rating depends on the current rating of the Credit and not the past ratings history. Obtaining in period $\mathrm{t}+2$ distribution states given $\mathrm{t}$ period distribution,

$X_{t+2}=X_{t+1} P=X_{t} P^{2}$

In case of time homogeneous and generally,

$X_{t+\mathrm{m}}=X_{t} P^{m}$.

In non-homogeneous cases,

$X_{t+2}=X_{t+1} P_{t+1}=X_{t} P_{t} P_{t+1}$

and

$X_{t+m}=X_{t} P_{t} P_{t+1}, \ldots, P_{t+\mathrm{m}-1}$

\section{5 Parameters of Markov Chain Transition Estimation}

Transition probabilities estimation in a Markov chain which is simple in a manner which is straightforward is done by counting from one state to another the changes that occur in a sample period. early papers by Anderson and Goodman (1957), Chatfield(1973) and Good- man(1957) investigated the problem of estimation and inference. Single period transition matrix is given by; 
$p=\left(\begin{array}{cc}P_{11} & 1-p_{11} \\ 1-p_{22} & P_{22}\end{array}\right)$

the number of times is given by nij and in $\mathrm{N}$ sample size $\mathrm{n}$, there is migration from state $\mathrm{i}$

to state $\mathrm{j}$. Markov process of likelihood function is given by;

$\ln L(P \mid 1-$ period data $)=n_{11} \ln p_{11}+n_{12} \ln \left(1-p_{11}\right)+n 21 \ln \left(1-p_{22}\right)+n_{22} \ln p_{22}$

The maximum likelihood estimators with respect to parameters p11 and p22 which are un- known are given by;

$\tilde{p}_{11}=n_{11} /\left(n_{11}+n_{12}\right) \tilde{p}_{22}=n_{22} /\left(n_{22}+n_{21}\right)$

Observing one step transitions, these estimators are appropriate for k-state cases. In two period transition data, estimating p11 and p22 in time homogeneous Markov chain is given by;

$$
p(2)=\left(\begin{array}{cc}
p_{11}(2) & 1-p_{11}(2) \\
1-p_{22}(2) & p_{22}(2)
\end{array}\right)=\left(\begin{array}{cc}
p_{11}^{2}+\left(1-p_{11}\right) & 1-\ldots \\
1-\ldots & p_{22}^{2}+\left(1-p_{11}\right)\left(1-p_{22}\right)
\end{array}\right)
$$

Letting state 1 observations to be denoted by $n_{11}$ (2) and moving from 1 to 2 be $n_{12}$ (2), two period transition loglikelihood estimation $p_{11}$ and $p_{22}$ data is

$$
\ln L(P / 2-\text { period data })=n_{11}(2) \ln p_{11}(2)+n_{12}(2) \ln \left(1-p_{11}(2)+n_{21}(2) \ln \left(1-p_{22}(2)\right)+n_{22}(2) \ln p_{22}\right.
$$

\section{Results}

Financial institutions estimate Markov transitional models basing on historical data. How- ever, one can obtain alternative sources for credit rating transition matrices. major rating agencies like Moody's and Standard and Poor's publish regularly transition matrices. Other vendors who also make use of transition matrices include KMV, Risk Metrics and Kamakura Inc. unfortunately, there is a laxity in reporting standards and often reporting is done with no underlying size of the data indicated or risk categories asset distribution. For accurate estimation, large data samples are vital since most credit transitions are events of low probability. Secondary data sets assembled to demonstrate the usefulness of the technique above include;

\section{1 Sovereign debt}

The Secondary data obtained from Standard and Poor's (2003) where there is adequate detail provided. Transition Matrices are calculated from overlapping Cohorts e. g from the 15 year sample, the cohorts are 115 year, 214 year, 315 year etc and the eight transition categories of default included recorded. There is careful definition of default in sovereign debt since some of the debt to the Creditors are serviced often after default. The transition rates for one year, three year and seven year are tabulated as below;

\section{Sovereign Debt 1995-2012}

\begin{tabular}{|l|l|l|l|}
\hline Transitions & Chi Square & DF & P-value \\
\hline 1,3 & 18.33 & 50 & 1.000 \\
\hline $1,3,5$ & 45.32 & 89 & 1.000 \\
\hline $1,2,5,7$ & 86.14 & 148 & 1.000 \\
\hline
\end{tabular}

No rejection of specification from time homogeneous Markov. The sample sizes here are below 100 hence the result.

\section{2 Municipal Bonds}

The Secondary data is from Standard and Poor's 2011 and consists of information from 1996 to 2010 from eight categories of rating municipal bonds. The reporting is done on average 1 year, 2 year and 15 year transitional matrices. The run tests are based on data from 1 year and 2 years, 1, 2, 3 years, etc up to the full data set i. e 1, 2, 3 up to 15 year transitions and tabulated as below;

\section{Municipal Bonds 1996-2010}

\begin{tabular}{|l|l|l|l|}
\hline Transitions & LR Statistic & DF & $\operatorname{Pr}\left(X^{2}>L R\right)$ \\
\hline 1,2 & 43.96 & 50 & 0.815 \\
\hline $1,2,3$ & 93.33 & 99 & 0.742 \\
\hline 1 to 4 & 155.2 & 148 & 0.427 \\
\hline 1 to 5 & 238.6 & 197 & 0.122 \\
\hline 1 to 6 & 374.9 & 246 & 0 \\
\hline 1 to 7 & 527.8 & 295 & 0 \\
\hline 1 to 8 & 715.7 & 344 & 0 \\
\hline 1 to 9 & 973.6 & 393 & 0 \\
\hline 1 to 10 & 1108 & 442 & 0 \\
\hline 1 to 11 & 1568 & 491 & 01 to 12 \\
2057 & 540 & 0 & \\
\hline 1 to 13 & 2491 & 589 & 0 \\
\hline
\end{tabular}


Asymptotic Properties of the Markov Chain Model method of finding Markov chains Generators of..

\begin{tabular}{|l|l|l|l|}
\hline 1 to 14 & 2867 & 638 & 0 \\
\hline 1 to 15 & 3178 & 687 & 0 \\
\hline
\end{tabular}

Up to a period of 5 Years, the Markov chain is adequate in transition ratings specification. The model is reliable for one year, two year, three year and sufficiently 4 year transitions and from 5 years, it begins to fail. This implies that in practise, rating transitions for municipal bond is described for periods reasonable by timehomogeneous models which are simple but should be updated after every four years.

\section{3 Commercial Paper}

The secondary data was obtained from a study done by Moody in 2010. Defaults in commercial paper are rare although some migration or transition rating categories may be seen in P-1, P-2, P-3 and NP where P denotes Prime. No prime rating should ever default according to Moody. Commercial papers are usually short term assets unlike Municipal Bonds and are examined in 30, 60, 90, 120, 180, 270 1nd 365 days. There is no problem experienced in the $150,210,240,300$ and 330 days powers of $\mathrm{P}$ missing since expected patterns are experienced are experienced up to 180 days data transitions and with a time span increase, transitions also move from initial state and probably to default. However, Commercial paper is seldom extended for long periods since for 270 days and 365 days, the migration from NP to Default is zero. The category WR(Withdrawn) is experienced in Commercial paper since the Commercial Paper is not rolled over hence resulting in the size of the asset becoming negligible or loss of interest in the Market offering. However, this is not a symbol of Creditworthiness decline or default and normally it is treated as censored. Illustrating the method, calculation is done basing on the increase in period number and results tabulated as below.

\section{Commercial Paper 1982-2009}

\begin{tabular}{|l|l|l|l|}
\hline Transition & Chi Square & DF & P-Value \\
\hline 1,2 & 15.95 & 16 & 0.535 \\
\hline $1,2,3$ & 19.83 & 32 & 0.979 \\
\hline $1,2,3,4$ & 24.78 & 48 & 0.999 \\
\hline 1 to 4,6 & & - & \\
\hline
\end{tabular}

Calculations based on Moody's (2010). The time scale is 30 days and the Model works for a sixmonths period relevant for applications. No evidence against the specification of Markov has been noted and the sample size here is big hence the results.

\section{Conclusion}

In most areas of portfolio management in bank risk management and supervision, Markov model which is time homogeneous is widely used. This model can describe simply the stochastic process of asset risk. for time homogeneity hypothesis, I propose a likelihood ratio test. Due to convenience of re-parameterization, to compute the test is simple and just requires restricted model numerical estimation $(K-1)^{2}$-a parameter problem where risk categories number is denoted as $K$. The test can be applied on the data collected by Banks or rating agencies. I recommend that the test be used as an interpretation to determine whether or not particular period transitions are Markov chains model. I do not recommend putting the test into use to determine the underlying process whether is Markovian since the prediction would lead to defaults in everything. I believe that most transitions can be Markovian model for several years. For example on an annual scale of up to 5 years, Municipal Bond ratings description can be time homogeneous Markov model. I recommend that a different model be used for larger transition period. I conclude that for practical use, the transition Matrix estimated should be regularly updated and then the forecast used for up to 5 years. For commercial paper, the Markov model which is time homogeneous can be described adequately on a time scale which is 30 days for six periods at least. Since the data is large, the result is fair although 30 day transitions period are not common. In Sovereign debt application, the specification of Markov on an annual scale transitions cannot be rejected since the sizes of the sample are small. This is because there is no indication that there will be an improvement of the Markov Model and also on the other hand, there is no indication that the Model works well because simply in these transitions which are rare, the information is very limited.

\section{References}

[1]. Bangla A., F. Diebold knonimus, C. Schagen and T. Schuermann (2002), "Ratings Migration and the Business cycle with applications to Credit portfolio stress testing".

[2]. Chatfield C. "Statistical Inference regarding Markov Chain Models; Applied Statistics

[3]. 22:1 (1973), 7-20

[4]. Karlin, S and Taylor, H. M. A first course in Stochastic process second edition. Academic Press New York 1975.

[5]. Basel Committee on Bank Supervision (June 2004), "International Convergence of Capital measurement and Capital Standards: A revised framework", Bank for international settlement.

[6]. Berthault, D. Hamilton, L. Carty, "Commercial paper defaults and Rating transitions, 
Asymptotic Properties of the Markov Chain Model method of finding Markov chains Generators of..

[7]. 1972-2000."

[8]. Standard and Poor's (2013), "2012 Defaults and rating transitions data for rated sovereigns"

[9]. Billingsley P. "Statistical methods in markov chains", Ann. Math. stat., 32(1951), 12-40

[10]. Thomas, L. C, D. B Edelman and J, N Crook(2002). Credit Scoring and its applications, SIAM, Philadelphia. 